\title{
Sweet Taste Preference in Women Smokers: Comparison With Nonsmokers and Effects of Menstrual Phase and Nicotine Abstinence
}

\author{
CYNTHIA S. POMERLEAU, ${ }^{1}$ ANNE W. GARCIA, ADAM DREWNOWSKI AND OVIDE F. POMERLEAU \\ Department of Psychiatry Behavioral Medicine Program, Division of Kinesiology \\ and School of Public Health, University of Michigan, Ann Arbor, MI
}

Received 22 August 1991

\begin{abstract}
POMERLEAU, C. S., A. W. GARCIA, A. DREWNOWSKI AND O. F. POMERLEAU. Sweet taste preference in women smokers: Comparison with nonsmokers and effects of menstrual phase and nicotine abstinence. PHARMACOL BIOCHEM BEHAV 40(4) 995-999, 1991. - Cigarette smokers weigh less than comparably aged nonsmokers, and many gain weight following cessation. Though some evidence suggests that nicotine reduces food intake, with a selective effect on sweet-tasting foods, the issue remains unresolved. In the current study, 64 women ( 20 smokers, 26 never-smokers, and 18 ex-smokers) were tested for sweet taste preference; 9 of these smokers were studied under conditions of both ad lib smoking and overnight abstinence, in three hormonally verified menstrual phases. 1) Although no overall differences were detected in taste preference among the three groups, significantly more smokers than nonsmokers preferred the higher sucrose concentrations. 2) No significant differences due to menstrual phase were observed. 3) Although preference ratings did not differ significantly between overnight abstinence and ad lib smoking, a subset of smokers who preferred higher sucrose concentrations rated their preference for the solutions significantly higher during the ad lib smoking sessions. Our findings suggest that smoking and nonsmoking women differ with respect to taste preference and that, at least in a subset of female smokers, preference is affected by nicotine abstinence/acute dosing.
\end{abstract}

Abstinence Ex-smokers Menstrual Nicotine Never-smokers Smokers Sweet taste

CIGARETTE smokers have repeatedly been shown to weigh less than comparably aged nonsmokers, and many smokers who quit smoking gain three to five $\mathrm{kg}$ in the year following cessation (5, $13,20,26)$. Though the reasons for this phenomenon are not fully understood, a number of studies have suggested that nicotine administration reduces food intake (14), with a selective effect on sweet-tasting foods $(6,8-10)$. Perkins et al. (17) have reported that hedonics of sweet taste are reduced in smokers as compared with nonsmokers. These investigators found no effects of acute nicotine administration upon taste hedonics, however; Gilbert and Pope (4) likewise did not observe any shift in preference for sweet foods following abstinence. There is even evidence in the animal literature to suggest that chronic nicotine exposure enhances appetite for sucrose (12). Thus the extent to which postcessation weight gain can be accounted for by changes in specific taste preferences (and consequent changes in types of foods consumed) remains unclear.

In an effort to extend our understanding of these issues, we studied 64 normal and obese women ( 20 smokers, 26 neversmokers, and 18 ex-smokers) who participated in a test of sweet taste perception and preference. Because sweet taste preference may also be influenced by menstrual phase $(18,24)$, a subset of 9 smokers was studied under conditions of both ad lib smoking and overnight abstinence in three hormonally verified phases of the menstrual cycle.

\section{Subjects}

METHOD

A community sample of 64 healthy women, not on medications, not pregnant or lactating, and not under treatment for obesity or eating disorders, was assembled as follows: 1) Fiftyeight women between the ages of 19 and 45 years old, unselected for smoking status, were recruited to the third author's laboratory to participate in various studies assessing taste perception and preference. The sample included both lean and obese subjects, with leanness being defined as weight below the 50th percentile for age decade and obesity as weight in excess of the 85th percentile for age decade, based on NHANES II data (16). Subjects were classified according to self-report as current smok-

\footnotetext{
${ }^{1}$ Requests for reprints should be addressed to Cynthia S. Pomerleau, Ph.D., Behavioral Medicine Program, University of Michigan Department of Psychiatry, Riverview Building, 900 Wall Street, Ann Arbor, MI 48105.
} 
ers (15 or more cigarettes/day), never-smokers, or ex-smokers; three subjects who smoked only 2 cigarettes a day were excluded from the analysis. 2) Nine normal-weight women smokers [body mass index $\left(\mathrm{kg} / \mathrm{M}^{2}\right)<27 ; 15$ or more cigarettes/day] between the ages of 20 and 39 were asked to participate in tests of taste perception and preference as an adjunct to a larger study of menstrual symptomatology, abstinence symptomatology, nicotine intake, and basal levels of cortisol and MHPG in three different menstrual phases under conditions of ad lib smoking and overnight abstinence (19), conducted in the fourth author's laboratory. Additional inclusion/exclusion criteria for these subjects were that they be: a) not using oral contraceptives or IUDs; b) not pregnant or at risk for becoming pregnant; c) free of complaints or history of treatment for PMS; and d) menstruating on a regular basis, with monthly cycles averaging between 26 and 35 days [see (19)]. The final combined database included 20 current smokers, 26 never-smokers, and 18 ex-smokers.

\section{Procedure}

Each subject in this study was exposed to a test used extensively by the third author (2) to assess responses to five different concentrations of sugar in water: $2 \%, 4 \%, 8 \%, 16 \%$, and $32 \%$. Each subject was asked to taste (less than one ounce) of each solution twice, for a total of 10 samples. Subjects rated the samples on a scale from 1 to 9 in terms of preference (dislike extremely to like extremely) and perception of sweet taste (not at all sweet to extremely sweet). Prior to sampling and between samples, the subject rinsed out her mouth with water. The samples were arranged in a random order at each of the laboratory sessions to avoid an order effect. To rule out satiation as a confounding factor, the protocol required spitting out, rather than swallowing, both the sample itself and the rinse water.

The nine subjects who participated in the menstrual phase/ smoking abstinence study were studied twice during each of three menstrual phases known to vary in terms of ovarian steroid milieu and menstrual symptomatology: 1) The early follicular (EF) phase, during which most women are in menses and when estrogen and progesterone are usually low, was defined as Day 2 through Day 5; Day 1 was avoided because of the potential confounding effect of dysmenorrhea. 2) The mid- to late follicular (M/LF) phase was the time period following menses and prior to the preovulatory luteinizing hormone (LH) surge; subjects were scheduled between Day 6 and Day 11. This phase was chosen because it was regarded as likely to be affectively and behaviorally "neutral" (21). 3) The mid- to late luteal phase (LL) was defined as the 4 days prior to menses, when estrogen and progesterone levels are beginning to drop and "premenstrual" symptoms tend to occur. A home test kit developed to aid in achieving or avoiding pregnancy (either OvuQUICK, manufactured by Monoclonal Antibodies, San Diego, CA; or Clearplan Easy, manufactured by Whitehall Laboratories, New York, NY) was used to pinpoint the LH surge, which precedes ovulation by 24 to 36 hours and the onset of next menses by approximately 14 days; subjects were scheduled accordingly. To verify that the cycle under investigation was ovulatory, subjects were asked to bring first morning urine samples to the lab on the 7th9th days following the LH surge, and a qualitative assay of pregnanediol glucuronide, a progesterone metabolite, was conducted.

Study sessions were conducted at midday (sometime between 10:00 a.m and 1:30 p.m; standard for each subject) on two consecutive weekdays (ad lib smoking vs. overnight abstinence sessions) in each of the three identified menstrual phases. For three of the six sessions, once in each menstrual phase, the subject smoked ad lib prior to that day's session. For the other three
TABLE 1

BASELINE DATA (MEANS \pm S.D.)

\begin{tabular}{ccccc} 
& & Menstrual & Never- & Ex- \\
N & Smokers & Study Subset & Smokers & Smokers \\
& 20 & $(9)$ & 26 & 18 \\
\hline
\end{tabular}

\begin{tabular}{lllll} 
Age & $31.1 \pm 7.4 \dagger$ & $(27.2 \pm 4.1)$ & $34.6 \pm 5.5^{*}$ & $39.2 \pm 4.7^{* \dagger}$ \\
BMI & $26.5 \pm 6.5$ & $(22.4 \pm 2.6)$ & $27.9 \pm 8.2$ & $29.9 \pm 6.4$ \\
\hline
\end{tabular}

$* p<0.05, \dagger p<0.001$.

sessions, the subject was asked to abstain from smoking for twelve hours preceding the session. At the beginning of each session, subjects were tested for expired carbon monoxide with the VITALOGRAPH-EC50 monitor to determine compliance with the abstinence requirement. On nonsmoking days, values above $15 \mathrm{ppm}$ would have disqualified subjects for that day's session. Compliance was subsequently verified by nicotine assay. To minimize the impact of possible order effects, subjects were randomly assigned to one of two smoking status sequences (ad lib smoking day first vs. abstinence day first) and one of three menstrual phase sequences (EF first, M/LF first, LL first; total of six possible sequences). Blood sampling occurred periodically via an indwelling catheter inserted into a forearm vein. Nicotine levels were assayed using an HPLC method (11); estradiol and progesterone were analyzed using RIA techniques $(3,16)$. Taste testing was conducted after all data for the main study had been collected and the catheter had been removed, by personnel trained by the third author and his staff (to ensure test administration conditions identical to those maintained in his laboratory). On smoking days only, to standardize the nicotine deprivation period, subjects smoked about 15 minutes prior to taste testing.

Data were analyzed using ANOVAs with smoking status as a grouping factor and sucrose concentration as a repeated measure. For the menstrual/abstinence study, repeated-measures ANOVAs, with 2 levels of smoking (ad lib smoking and overnight abstinence), 3 levels of menstrual phase (EF, M/LF, and LL), and 5 levels of sucrose concentration were conducted. Post hoc tests were carried out as appropriate. Chi-square analyses were carried out to determine whether the proportion of sweettaste preferrers (those whose scores on the $16 \%$ and $32 \%$ concentrations exceeded scores on the $2 \%$ and $4 \%$ concentrations) differed significantly among groups.

\section{RESULTS}

Significant age differences among the groups were detected, $\mathrm{F}(2,61)=2156.52, p<0.0001$. Post hoc analysis using the $t$-test for differences among several means (1) revealed that the exsmokers were significantly older than both the nonsmokers $(p<0.05)$ and the smokers $(p<0.001)$; smokers and nonsmokers did not differ significantly in age. No significant group differences emerged for BMI.

Preliminary analyses revealed no differences in taste behavior based on BMI, so data for normal-weight and obese subjects were pooled. Table 1 shows baseline data for the three groups of smokers, and for the subgroup that participated in the menstrual phase/abstinence study.

As shown in Fig. 1, all groups and subgroups showed an orderly and highly significant dose-response curve for perception of sweet taste [overall, $\mathrm{F}(4,244)=235.51, p=0.000]$. No significant group or interaction effects were detected. 


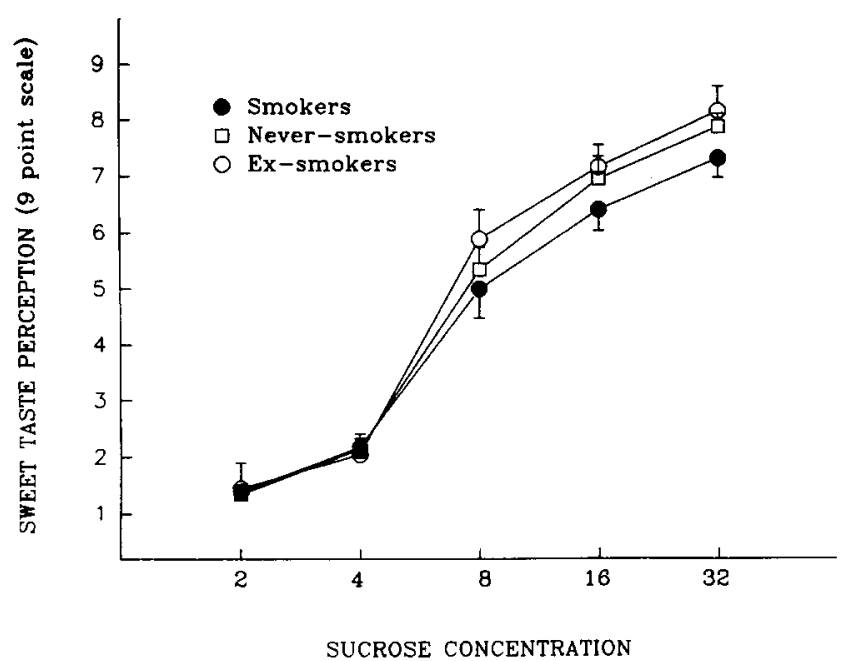

FIG. 1. Perception of sweet taste for smokers $(n=20)$, ex-smokers $(n=$ 18 ), and never-smokers $(n=26)$ (mean \pm s.e.m.).

\section{Group Differences in Sweet-Taste Preference}

Since no significant effect for menstrual phase was uncovered (see below), and since other subjects included in the database had been tested without reference to menstrual phase, a mean of ratings for the three ad lib smoking sessions was computed to provide the datapoints for these nine subjects in subsequent analyses of group differences. Figure 2 shows overall preference patterns for the three groups. A 2-way ANOVA (3 smoking status groups $\times 5$ concentrations) revealed no significant main or interaction effects.

Previous experience with the taste testing procedure has led to the identification of two groups of people-sweet-taste preferrers (those who rate the two highest concentrations more favorably than the two lowest) and nonpreferrers (those who do not) (23). Preferrers comprised $45 \%$ of the smokers $(9 / 20), 33 \%$ of the ex-smokers $(6 / 18)$, and $8 \%$ of the never-smokers $(2 / 26)$; a chi-square test indicated a significant relationship between sweet

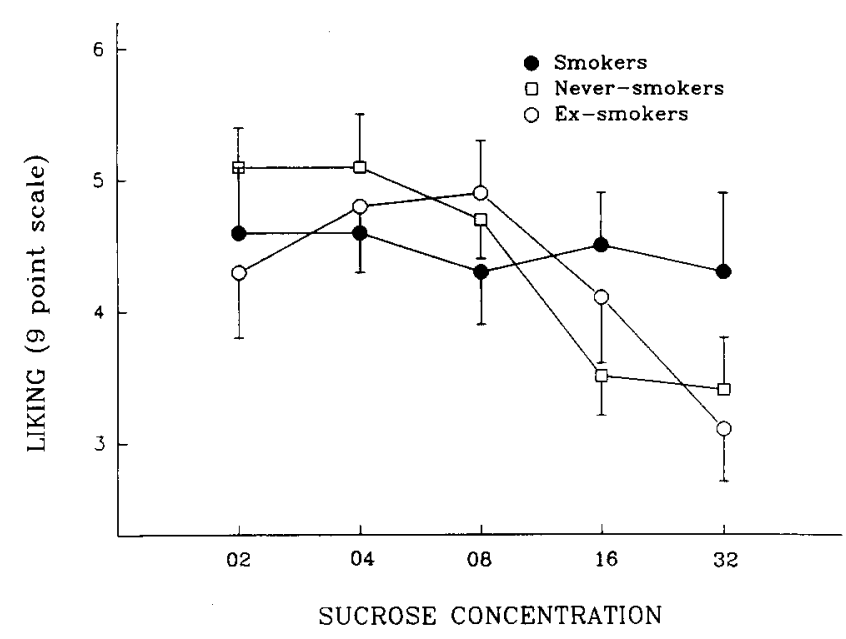

FIG. 2. Sweet taste preference patterns for smokers $(n=20)$, ex-smokers $(n=18)$, and never-smokers $(n=26)$ (mean \pm s.e.m.). taste preference and smoking status (chi-square $=8.65, p=0.013$ ).

Effects of Menstrual Phase and of Overnight Abstinence Upon Sweet Taste Preference

All subjects in the menstrual/abstinence study succeeded in meeting the criteria for overnight abstinence, as measured by $\mathrm{CO}$ and subsequently verified by plasma nicotine levels of $<10 \mathrm{ng} /$ $\mathrm{ml}$. Some difficulties in predicting the LL phase occurred; of the total of $18 \mathrm{LL}$ sessions ( $2 \mathrm{LL}$ sessions for each of 9 subjects), 4 subsequently proved to have been scheduled 1-3 days earlier than intended, and 1 had to be run shortly after the onset of menses because insurmountable logistical problems prevented rescheduling during the next month. Consistent with the fact that several subjects were tested early, means for LL progesterone $(5.7 \pm 2.0 \mathrm{ng} / \mathrm{ml}$ for the ad lib smoking day; $6.3 \pm 2.5 \mathrm{ng} / \mathrm{ml}$ for the abstinence day) exceeded the expected values for Day -4 through onset of menses (usually $<2 \mathrm{ng} / \mathrm{ml}$ ); all other progesterone means and all estradiol means were in the expected range. As anticipated, there was a significant main effect of phase for progesterone and a trend towards a significant phase effect for estradiol; no smoking status or interaction effects were detected (19).

The taste test results were analyzed using a 3 (menstrual phase) $\times 2$ (smoking condition) $\times 5$ (sugar water concentration). No significant main or interaction effects emerged from this analysis. A separate analysis including only the five sweettaste preferrers was conducted. As expected (since subjects were selected for uniformity along this dimension), it revealed a significant main effect for concentration, $\mathrm{F}(4,16)=48.0, p=0.000$. There was also a significant main effect for ad lib smoking vs. abstinence, $\mathrm{F}(1,4)=8.00, p=0.047$, but no significant main effect for menstrual phase or any interaction. No such pattern emerged from an analysis of the four nonpreferrers. Figure 3 shows preference patterns for the two preference groupings among the nine smokers included in this analysis. For preferrers, nonpreferrers, and the group as a whole, perception of sweet taste showed significant increases as a function of concentration [respectively, $F(4,16)=92.11 ; F(4,12)=214.33 ; F(4,32)=249.53$; for all, $p=0.000$ ] but no significant main effects for phase or smoking and no significant interactions.

\section{DISCUSSION}

Consistent with the findings of Perkins et al. (17), an orderly dose-response curve was observed for perception of sweet taste in the group as a whole and in all subgroups. Thus no distortions in perception appear to be associated with smoking or a history of having smoked.

\section{Group Differences in Sweet Taste Preference}

Although no overall differences were detected among taste preference curves for the three groups, significantly more smokers than nonsmokers showed a preference for the higher sucrose concentrations-possibly indicating that a higher degree of stimulation was required in smokers to produce satisfaction. Prospective studies would be required to determine whether the preference preceded smoking acquisition, or whether it rather resulted from the central or peripheral effects of chronic smoking/nicotine administration. The apparent persistence of these effects in a substantial percentage of ex-smokers tends to suggest that a preference for sweeter-tasting food may have preceded smoking initiation and indeed, may serve as a marker of susceptibility to smoking; alternatively, chronic nicotine use may produce irreversible changes 

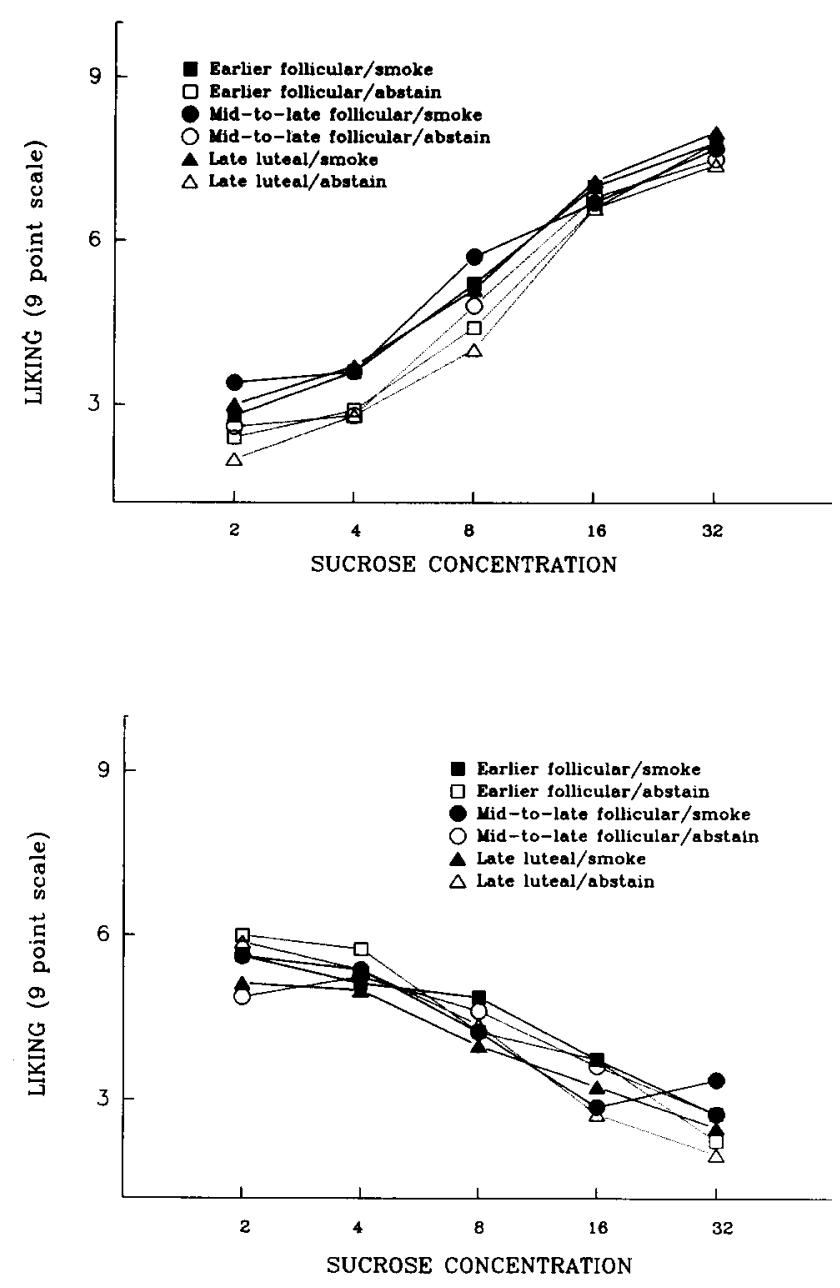

FIG. 3. Sweet taste preference patterns in 5 "preferrers" (top) and 4 "nonpreferrers" (bottom) under conditions of ad lib smoking and overnight abstinence for each of 3 menstrual phases (mean \pm s.e.m.; $n=9$ ).

in sweet taste preference. The persistence in ex-smokers also renders it unlikely that the observed differences are attributable to the significant between-group age differences, since the nonsmokers were older than the smokers and younger than the exsmokers.

\section{Effects of Menstrual Phase and of Overnight Abstinence Upon Sweet Taste Preference}

No significant differences due to menstrual phase were observed. Because of our difficulties in predicting the $\mathrm{LL}$ phase, as confirmed by higher mean progesterone levels than expected, we may have missed capturing effects specifically attributable to "premenstrual" factors. Furthermore, our small sample may have been inadequate to reveal phase differences if they existed.

Consistent with the findings of Perkins et al. (17), no significant differences in taste preference between overnight abstinence and ad lib smoking were observed. When the subjects were divided into preferrers and nonpreferrers, however, the former rated their overall preference for the solutions significantly higher during the ad lib smoking sessions than during the nonsmoking sessions. Thus, after a brief abstinence from smoking, there was a preference shift such that, for example, an $8 \%$ concentration of sucrose was required, among these "sweet tooth" subjects, to produce a preference rating similar to ratings of the $2 \%$ and $4 \%$ concentrations during ad lib smoking. If differences in preference between abstinence and ad lib smoking persist over time, then such smokers might tend to eat sweeter foods following smoking cessation in order to achieve an equivalent degree of satisfaction. Since comparable abstinence-related differences in perception of sweet taste were not detected, it seems unlikely that the observed differences in preference were attributable to the acute effects of smoking on taste perception; a larger sample, however, would be required to rule out this possibility. No such orderly effects were observed in the nonpreferrers, but again, a larger sample would be required to achieve adequate power to reject the null hypothesis definitively.

\section{Conclusion}

Our findings overall provide evidence of differences between smoking and nonsmoking women with respect to taste preference and suggest that, at least in a subset of female smokers who prefer sweeter-tasting solutions, preference is affected by nicotine abstinence/acute nicotine dosing. Although it cannot be determined from our study whether our findings can be generalized to male smokers, the fact that women smokers tend to be more concerned about weight gain than their male counterparts $(7,21)$, combined with the fact that the prevalence of smoking in American women has shown a much shallower decline than in men (25), underscores the particular importance of such research in female samples. It is to be hoped that studies such as the present one will contribute to the identification of factors specific to smoking in women that could be used as the basis for the development of more effective, targeted treatment strategies.

\section{ACKNOWLEDGEMENTS}

This investigation was supported in part by grants to the first and second authors (University of Michigan Psychiatry Research Committee) and the fourth author (National Cancer Institute, CA-42730; and NIDA DA-06529). We wish to thank Susan M. Basson, B.S. and Karen A. Flessland, B.S. for their invaluable assistance in carrying out this project.

\section{REFERENCES}

1. Bruning, J. L.; Kintz, B. L. Computational handbook of statistics. 3rd ed. Glenview, IL: Scott, Foresman and Company; 1987.

2. Drewnowski, A.; Schwartz, M. Invisible fats: Sensory assessment of sugar/fat mixtures. Appetite 14:203-217; 1990.

3. England, B. G.; Niswender, G. D.; Midgley, A. R. Radioimmunoassay of estradiol 17 beta without chromatography. J. Clin. Endocrinol. Metab. 38:42; 1974.

4. Gilbert, R. M.; Pope, M. A. Early effects of quitting smoking. Psychopharmacology (Berlin) 78:121-127; 1982.

5. Grunberg, N. E. Behavioral and biological factors in the relation- ship between tobacco use and body weight. In: Katkin, E. S.; Manuck, S. B., eds. Advances in behavioral medicine. vol. 3. Greenwich CT: JAI Press; 1986:97-129.

6. Grunberg, N. E. The effects of nicotine and cigarette smoking on food consumption and taste preferences. Addict. Behav. 7:317-331; 1982.

7. Grunberg, N. E. Inverse relationship between tobacco use and body weight. In: Kozlowski, L. T.; Annis, H .M.; Cappell, H. D.; Glaser, F. B.; Goodstadt, M. S.; Israel, Y.; Kalant, H.; Sellers, E. M.; Vingilis, E. R., eds. Research advances in alcohol and drug 
problems, vol, 10. New York: Plenum Press; 1990:273-316.

8. Grunberg, N. E.; Bowen, D. J.; Winders, S. E. Effects of nicotine on body weight and food consumption in female rats. Psychopharmacology (Berlin) 90:101-105; 1986.

9. Grunberg, N. E.; Winders, S. E.; Popp, K. A. Sex differences in nicotine's effects on consummatory behavior and body weight in rats. Psychopharmacology (Berlin) 91:221-225; 1987.

10. Hall, S. M.; McGee, R.; Tunstall, C.; Duffy, J.; Benowitz, N. Changes in food intake and activity after quitting smoking. J. Consult. Clin. Psychol. 57:81-86; 1989.

11. Hariharan, M.; Van Noord, T.; Greden, J. F. A HPLC method for the routine simultaneous determination of nicotine and cotinine in plasma. Clin. Chem. 34:724-729; 1988.

12. Jias, L. M.; Ellison, G. Chronic nicotine induces a specific appetite for sucrose in rats. Pharmacol. Biochem. Behav. 35:489-491; 1990.

13. Klesges, R. C.; Meyers, A. W.; Klesges, L. M.; LaVasque, M. E. Smoking, body weight, and effects on smoking behavior: A comprehensive review of the literature. Psychol. Bull. 106:204-230; 1989.

14. McNair, E; Bryson, R. Effects of nicotine on weight change and food consumption in rats. Pharmacol. Biochem. Behav. 18:341-344; 1983.

15. Najjar, M. F.; Rowland, M. Anthropometric reference data and prevalence of overweight, United States, 1976-80. Vital and health statistics, series 11, No. 238. DHHS publication No. (PHS) 871688, Public Health Service. Washington, DC: U.S. Government Printing Office; 1987.

16. Niswender, G. D. Influence of the site of conjugation on the specificity of antibodies to progesterone. Steroids $22: 413 ; 1973$.

17. Perkins, K. A.; Epstein, L. H.; Stiller, R. L.; Fernstrom, M. H.; Sexton, J. E.; Jacob, R. G. Perception and hedonics of sweet and fat taste in smokers and nonsmokers following nicotine intake. Pharmacol. Biochem. Behav. 35:671-676; 1990.
18. Pliner, P.; Fleming, A. S. Food intake, body weight, and sweetness preferences over the menstrual cycle in humans. Physiol. Behav. 44:109-116; 1983

19. Pomerleau, C. S.; Garcia, A. W.; Pomerleau, O. F.; Cameron, O. G. The effects of menstrual phase and nicotine abstinence on nicotine intake and on biochemical and subjective measures in women smokers. In review.

20. Pomerleau, O. F.; Adkins, D.; Pertschuk, M. Predictors of outcome and recidivism in smoking-cessation treatment. Addict. Behav. 3:65$70 ; 1978$.

21. Schechter, D.; Bachmann, G. A.; Vaitukaitis, J.; Phillips, D.; Saperstein, D. Perimenstrual symptoms: Time course of symptom intensity in relation to endocrinologically defined segments of the menstrual cycle. Psychosom. Med. 51:173-194;1989.

22. Streater, J. A.; Sargent, R. G.; Ward, D. S. A study of factors associated with weight gain in women who attempt smoking cessation. Addict. Behav. 14:523-530; 1989.

23. Thompson, D. A.; Moskowitz, H. R.; Campbell, R. Effects of body weight and food intake on pleasantness ratings for sweet stimulus. J. Appl. Psychol. 41:77-83; 1976.

24. Tomelleri, R.; Grunewald, K. K. Menstrual cycle and food cravings in young college women. J. Am. Diet. Assoc. 87:311-315; 1987.

25. U.S. Department of Health and Human Services (USDHHS). Reducing the health consequences of smoking: 25 years of progress. A report of the Surgeon General. U.S. Department of Health and Human Services, Public Health Service, Centers for Disease Control, Center for Chronic Disease Prevention and Health Promotion, Office on Smoking and Health. DHHS publication No. (CDC) 898411. Washington, DC: U.S. Government Printing Office; 1989.

26. Williamson, D. F.; Madans, J.; Anda, R. F.; Kleinman, J. C.; Giovino, G. A.; Byers, T. Smoking cessation and severity of weight gain in a national cohort. N. Engl. J. Med. 324:739-745; 1991. 